\title{
CONVERGÊNCIA, PARA ONDE? UMA ANÁLISE DA DINÂMICA DE DISTRIBUIÇÃO DE RENDA PER CAPITA A PARTIR DO MODELO DE MISTURAS FINITAS.
}

\author{
Eva Yamila da Silva Catela* \\ Flávio de Oliveira Gonçalves ${ }^{\dagger}$
}

\begin{abstract}
Resumo
Diferentes teorias de crescimento implicam em diferentes padrões de distribuição de níveis de renda per capita entre as economias mundiais. A validação empírica de tais teorias pressupõe propriedades nos processos estocásticos nem sempre verificadas nos exercícios empíricos tradicionais de regressões de crescimento. Este trabalho busca estas propriedades e discute a existência de clubes de renda na economia mundial analisando a distribuição da renda per capita em 134 países durante o período 19702003. As conclusões mostram uma estratificação em três grupos, com path dependence sem lock in, isto é existe uma alta probabilidade de permanência do status quo.
\end{abstract}

Palavras-chave: Crescimento, Misturas Finitas, Convergência em Clubes

\begin{abstract}
Different growth theories implies in different patterns of per capita income distribution between countries. The empirical validation to these theories assumes some non-commonly verified stochastic processes properties. This paper seeks for theseis properties and discusses the existence of per capita income clubs in the world economy by analyzing the distribution of per capita income in 134 countries during the period 19702003. The conclusions show stratification in three groups, with path dependence and no lock in, that is, there is a high probability of maintenance of the status quo, while there exists a small probability of migration between groups.
\end{abstract}

Keywords: Growth, Finites Mixtures, Club Convergence JEL classification: C14, O40, O50.

\footnotetext{
* Professora da Faculdade de Ciências Econômicas da Universidade Federal de Santa Catarina. Endereço: Campus Universitário UFSC. Centro Sócio-Econômico, Departamento de Economia. Trindade, CEP: 88040-970. E-mail: evadasilvacatela@gmail.com

† Professor Adjunto da Faculdade de Ciências Econômicas da Universidade Federal de Paraná. Email: f.goncalves@ufpr.br
} 


\section{Introdução}

A pesquisa sobre a existência ou não de convergência entre rendas per capita nos países do mundo tem produzido uma grande quantidade de estudos, empíricos e teóricos. Nos modelos neoclássicos tradicionais de crescimento a principal conclusão diz respeito ao crescimento como um processo exógeno inexorável. Os retornos decrescentes do capital asseguram a convergência da renda entre países com mesmos fundamentos. A partir da década de 1980 foi observado que o comportamento da distribuição da renda nacional dos países é caracterizado pela existência de dois ou mais grupos que distinguem um mundo polarizado e sugere a existência de equilíbrios múltiplos. Neste sentido, o trabalho seminal de Baumol (1986) aponta a existência de clubes de convergência (OECD, economias centralmente planificadas e países em desenvolvimento) e demonstra que os padrões de convergência diferem entre estes grupos. A questão da multimodalidade na distribuição de renda foi logo salientada por Quah $(1996,1997)$, que estabelece que em 1990 o mundo estava dividido - como fato estilizado - em duas categorias: os ricos e os pobres. Outra contribuição no mesmo sentido é a de Bianchi (1997), que sugere que a defasagem entre países mais e menos desenvolvidos (distância entre os modos) se ampliou durante os anos 1980.

O trabalho de David $(2003,2007)$ questiona o fato de que o modelo de convergência explica o fenômeno econômico sem fazer referêencia aos legados do passado, ou seja, trata-se de um processo path-independent cuja dinâmica garante convergência para uma configuração de equilíbrio única e globalmente estável. O conceito de path dependence se relaciona, por sua vez, com um processo estocástico cuja distribuição assintótica evolui como conseqüência do seu processo histórico. Desta forma, as condições iniciais são importantes, porém não determinísticas de todo processo histórico vindouro.

Este trabalho determina o número de grupos em que podemos dividir os países do mundo de acordo com seus níveis de renda per capita a partir de uma estimação de densidade não paramétrica e do modelo de misturas finitas para a renda per capita (ajustada por paridade de poder de compra) de 134 países do mundo, no período entre 1970-2003, caracterizando esses grupos e suas dinâmicas. O comportamento desta distribuição fornece importantes conclusões sobre a existência de clubes de renda no mundo, sua conformação e a dinâmica intra-distribuição ao longo do tempo. Modelos estatísticos baseados em distribuições de mistura finita captam muitas propriedades específicas dos dados como multimodalidade, assimetria, curtose e heterogeneidade não observada Frühwirth-Schnatter (2006).

A utilização deste modelo permite fazer uma análise mais rica da distribuição de renda entre os países do mundo, informando-nos melhor sobre a dinâmica de catching up dos países relativamente mais pobres em relação aos mais ricos, da estagnação de certos países em armadilhas de pobreza, da ultrapassagem daqueles previamente mais ricos por alguns países pobres, da dinâmica de convergência em clubes com países membros convergindo juntos para certo nível de renda e divergindo de outros clubes diferentes. As principais conclusões do trabalho nos mostram a estagnação da renda dos países mais pobres desde a década de 70, a mudança de configuração da distribuição depois do fim do tratado de Bretton Woods, o distanciamento entre os grupos de renda, a convergência entre os países mais ricos e o aumento de heterogeneidade na classe de países intermediários. 
O trabalho é organizado como segue. Na seção dois é apresentado o debate da convergência versus divergência de renda e as características que determinam as diferenças de renda entre países, assim como o conceito de path dependence. Na seção três são apresentados e definidos os dados utilizados, assim como a função de densidade de Kernel para renda, como primeira aproximação para a análise. Na seção quatro é descrito o método empregado para a realização da análise empírica, o modelo de misturas finitas, assim como o teste econométrico usado para a análise do número de componentes da distribuição, o Teste LR. A seção cinco apresenta os principais resultados econométricos. Depois de determinar o número de componentes nos grupos de renda per capita, será analisada a evolução e a dinâmica inter-distribuição da renda, utilizando os dados de média e desvio padrão, e a composição dos grupos determinados. Para finalizar, são apresentadas as conclusões do trabalho, na seção sete.

\section{O debate da convergência}

Nos modelos neoclássicos de crescimento como o de Solow (1956), a taxa de crescimento da renda per capita é inversamente relacionada com seu nível inicial. O elemento chave que explica o resultado da convergência nestes modelos são os retornos decrescentes do capital reproduzível. Se as economias têm os mesmos parâmetros de preferências e tecnologia existe uma força que promove a convergência (Barro e Sala-I-Martin 1992). Desta forma, são ignoradas a inovação tecnológica e sua difusão, ou é assumido que a tecnologia é um bem público criado fora da esfera econômica e que os parâmetros não sofrem alteração no decorrer do processo histórico. O resultado fundamental do modelo neoclássico é a existência da convergência de renda entre países. A convergência beta absoluta é definida como a existência de uma relação negativa entre o nível alcançado de produto per capita e sua taxa de crescimento. Existe convergência absoluta $\beta$ se as economias pobres tendem a crescer mais rápido do que as economias ricas. Neste contexto, deve existir uma correlação negativa entre o nível inicial de renda e a taxa de crescimento subseqüente. Dada a hipótese de rendimento decrescente do capital, isto significa que os países pobres em capital (e renda) terão uma maior produtividade marginal do capital.

Este resultado contrasta com as implicações da primeira geração de modelos de crescimento endógeno, como o de Romer (1986). O ponto chave desses modelos é a ausência de retornos decrescentes de capital (em que capital inclui capital humano) com a conseqüência de inexistência da propriedade de convergência.

Muitos trabalhos examinaram a natureza do processo de crescimento dentro do modelo neoclássico e fora dele, apontando para a convergência (Baumol 1986, Barro 1991) ou para a persistência de diferenças na renda per capita entre países (Romer 1986, Lucas 1988). Como conseqüência destes trabalhos, porém, a controvérsia e o debate acerca de como medir e interpretar a convergência e o crescimento dos países aumentou. A falta de convergência entre países foi utilizada como evidência contra o modelo neoclássico e a favor dos novos modelos de crescimento endógeno. Sem retornos decrescentes, a poupança e o investimento podem gerar crescimento indefinidamente e as políticas públicas podem mudar a taxa de poupança e conseqüentemente a taxa de crescimento. 
Outros autores como Quah (1993) mostraram que o conceito de convergência é uma proposição acerca da dispersão da distribuição de renda (e a taxa de crescimento) entre países e um $\beta$ negativo no resultado da regressão do nível inicial de renda não implica necessariamente uma redução nesta dispersão. A dispersão nada nos diz acerca da "agitação" dentro da cross-section. Entender a dinâmica intra-distribuição pode nos informar melhor sobre o processo de catching up dos países pobres em relação aos ricos, a estagnação de países dentro de armadilhas de pobreza, países pobres ultrapassando os níveis de renda de países previamente mais ricos e a dinâmica de clubes de convergência, isto é, de grupos de paises que convergem para uma média conjunta e divergem de outros grupos de paises.

No inicio da década de noventa, distintos autores (Barro e Sala-I-Martin 1990, 1992, Mankiw et al. 1992) utilizaram uma nova definição de convergência, denominada convergência condicional, ${ }^{1}$ para demonstrar que o modelo neoclássico é consistente com a evidência empírica. Estes economistas argumentaram que o modelo neoclássico estabelecia que, se parâmetros tecnológicos, de preferências, ou institucionais diferem entre dois países, estes possuem estados estacionários diferentes.

A idéia básica é que quanto mais longe uma economia se localiza de seu próprio nível de estado estacionário, maior é a defasagem do estoque de capital reproduzível (físico e humano) por trabalhador e da eficiência técnica de seu nível potencial de longo prazo. Desta forma, o modelo prediz que a taxa de crescimento de uma economia está inversamente relacionada com a distância que a separa de seu próprio estado estacionário; países de baixo produto per capita têm a oportunidade de aproximar-se rapidamente daqueles de alto nível de produto per capita e se todos os países possuem o mesmo "estado estacionário" se produzirá uma equiparação no seu nível de bem estar, através de acumulação maior de capital e difusão de tecnologia desde países mais avançados tecnicamente para os menos avançados. Deve-se enfatizar, no entanto, a convergência de cada economia a seu próprio estado estacionário. Assim, o que se deve encontrar é a correlação parcial negativa entre crescimento e nível de produto inicial condicional ao estado estacionário. Como citado em Quah (1993), o simples sinal negativo da relação entre renda inicial e taxa de crescimento pode indicar apenas uma regressão para a média, ignorando todos os movimentos intra-distribuição e mudanças de posição no ranking de renda per capita entre os países.

Como apontado por Islam (2003), o conceito de convergência condicional é relacionado com a noção de "clubes de convergência". No caso de convergência incondicional, o equilíbrio para o qual todas as economias se dirigem é único. No caso de convergência condicional, cada economia se aproxima do seu próprio equilíbrio definido pelas suas condições iniciais. Empiricamente, procuram-se variáveis para controlar as características de cada país e encontra-se uma taxa global de convergência condicional. Em contraste, a idéia de clubes de convergência é baseada na existência de equilíbrios múltiplos. Um grupo de países pode-se aproximar a um equilíbrio particular se eles têm os atributos correspondentes ou se encontram numa localização inicial correspondente àquele equilíbrio. Isto produz convergência em clubes, ou regimes múltiplos Durlauf e Johnson (1995). Neste caso, as economias têm

\footnotetext{
${ }^{1}$ Só se todas as economias convergem para o mesmo steady state a $\beta$-convergência condicional e
} absoluta coincidem. 
um grau de dependência de suas condições iniciais, criando uma resistência a permanecer em seu grupo, porém, segundo seu processo histórico, algumas economias podem vencer esta resistência e ingressar em um grupo de renda mais alta (como no caso da Espanha, Irlanda, Coréia do Sul), ou mesmo serem rebaixadas a um grupo mais pobre (como os casos de desastre de crescimento observados na Argentina na metade do século e na Venezuela).

Vários modelos teóricos descrevem as forças econômicas que determinam à formação destes clubes apontando a perda de informação por trás dos exercícios empíricos de convergência condicional. Quah (1993), por exemplo, enfatiza que o fato de controlar por variáveis explicativas como capital humano ou características sociais leva ao pesquisador a concluir erroneamente que são essas características que determinam à posição econômica do país. Na visão tradicional, o pesquisador estima uma regressão cross-section observando o comportamento (condicional) da economia representativa, tendo como resultado uma taxa constante, globalmente estável, de convergência condicional, perdendo, assim, a multimodalidade que aparece na distribuição de renda dos países e a dinâmica intradistribuição.

Evans (1997) mostra que, ainda que as variáveis de controle expliquem $90 \%$ da variância do produto per capita no estado estacionário, o limite da probabilidade do estimador do coeficiente do produto inicial per capita é de aproximadamente a metade do valor verdadeiro. Levine e Renelt (1992) apontam que as regressões cross section não são robustas de acordo com o conjunto de variáveis de controle utilizadas.

Num caminho diferente de pesquisa, David (2003, 2007), mostra que o modelo de convergência é atemporal, no sentido de que tenta explicar o fenômeno econômico sem referência aos legados do passado. Neste sentido, tratase de um processo path independent, dado que a sua dinâmica garante convergência para uma configuração de equilíbrio única e estável. Ainda mais sendo que, nesta estrutura, os feedbacks positivos dominam a vida econômica. No limite, uma probabilidade positiva única é verificada para cada uma das configurações que podem ser assumidas.

$\mathrm{O}$ conceito de path dependence refere-se à propriedade dinâmica do processo distributivo, ou à relação entre a dinâmica do processo e o resultado ao qual ele converge. Neste caso, o processo não é capaz de ficar livre de sua história, sendo o resultado dependente do caminho que ele seguiu. Alternativamente, a propriedade de path dependence pode ser definida para a classe de processos estocásticos referindo-se não ao resultado, mas à natureza da distribuição de probabilidade que eventualmente vai governar os movimentos do sistema sob análise. O Teorema Central do Limite deixa de funcionar neste mundo e perturbações aleatórias, transitórias, podem exercer uma influência forte nos movimentos do sistema das raízes unitárias nas séries temporais).

Um sistema estocástico path dependent é um que possui uma distribuição assintótica que evolui como conseqüência do seu processo histórico. Nesta dinâmica não-ergódica, deve ser considerada a probabilidade da história se repetir num sentido estrito.

Este trabalho leva a sério a existência de diferentes padrões de crescimento na renda dos países, existência de armadilhas de pobreza e riqueza e path dependence. Existe um grande número de resultados teóricos que oferecem justificativa para a existência destas diferenças nos padrões de crescimento. A análise destes fatores excede os limites deste trabalho, mas podemos citar brevemente alguns destes, como, por exemplo, a instabilidade política (Alesina 
Tabela 1: Estatísticas descritivas do PIB per capita para o total de países da amostra(PPP, Chain, ano base 1996)

\begin{tabular}{cccccc}
\hline Ano & Obs. & Média & Erro Padrão & Mínimo & Máximo \\
\hline 1970 & 134 & 4866,47 & 4764,29 & 415,08 & 21111,26 \\
1975 & 134 & 5539,09 & 5357,68 & 489,36 & 20980,77 \\
1980 & 134 & 6304,96 & 6002,72 & 505,30 & 24048,65 \\
1985 & 134 & 6619,85 & 6372,54 & 461,96 & 24877,87 \\
1990 & 134 & 7373,69 & 7298,89 & 463,73 & 27515,26 \\
1995 & 134 & 7929,28 & 7926,34 & 485,97 & 29248,77 \\
2000 & 134 & 9077,03 & 9138,33 & 681,63 & 34364,50 \\
2003 & 134 & 9407,10 & 9376,19 & 682,54 & 34875,37
\end{tabular}

Fonte: Elaboração dos autores em base aos dados de PWT versão 6.2.

et al. 1996), a localização dos países (Moreno e Trehan 1997), a existência de livre comércio (Ben-David e Loewy 1998), o grau de intervenção do governo (Lee 1996), a instabilidade regional (Ades e Chua 1997), os conflitos sociais (Benhabib e Rustichini 1996), a distribuição do capital humano (Galor e Tsiddon 1997), a intensificação de capital (Dollar e Wolff 1994) e as diferenças em capacidades tecnológicas, como Nakajima (2003), Howitt e Mayer-Foulkes (2002) e Castellacci e Archibugi (2005).

A seguir são apresentados brevemente os dados de renda utilizados e se procede à realização de uma primeira análise não-paramétrica da distribuição da renda dos países do mundo.

\section{Estimação de densidade de Kernel}

Na tabela seguinte são apresentadas as estatísticas descritivas para o PIB per capita ajustado por paridade do poder de compra, para os anos que vão de 1970 a 2003, tomando como ano base 1996. O número de países incluídos na amostra é de 139. Os dados são da Penn World Table 6.2 (Heston et al. 2006).

A primeira característica que podemos observar é o aumento da média do PIB per capita, ao longo do período coberto pelo estudo. Entretanto, só uma análise intra-distribuição poderá nos informar se esse aumento corresponde a uma melhora na renda de todos os países ou só de uma parte deles. No caso do desvio padrão este também apresenta um importante aumento ao longo do tempo. A partir destas mudanças podemos inferir que pode ter ocorrido mudanças dentro da distribuição de renda per capita dos países.

A observação da distribuição é uma boa forma de começar a analisar os valores de preditores lineares que vamos utilizar posteriormente para cada grupo ou clube, na realização do teste de misturas finitas. Na seguinte figura são apresentadas três funções de densidade de Kernel, para os anos 1970, 1990 e 2003, com três quartos de amplitude ${ }^{2}$. A análise exploratória dos dados, em especial como se apresenta sua distribuição através de estimadores nãoparamétricos ajudam a fazer um diagnóstico da situação antes da estimação propriamente dita.

A avaliação dos padrões de dinâmica intra-distribuição, como, por exemplo, a possível evidência de polaridade ou multi-modalidade, é de extrema

\footnotetext{
${ }^{2}$ Quanto maior a amplitude, maior é a suavidade da curva, porém, também é maior a perda de informação.
} 


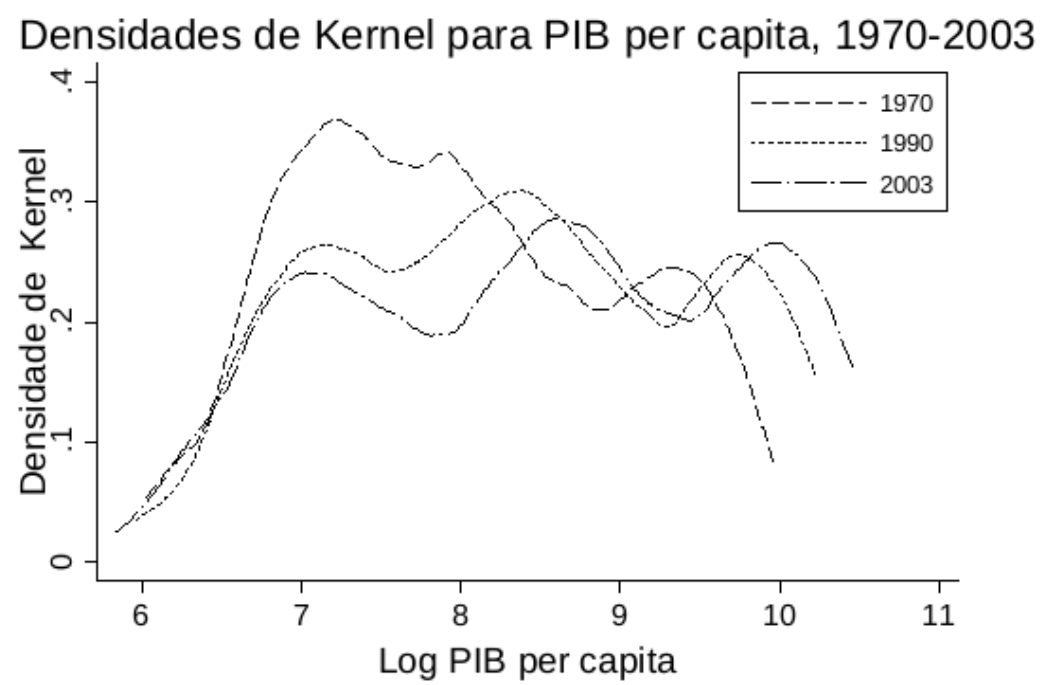

Fonte: Elaboração dos autores no Stata.

Figura 1: Função de Densidade de Kernel para 1970, 1990 e 2003

importância se a mesma implica uma melhora ou piora da situação de progresso tecnológico e o conseqüente crescimento econômico. Em termos teóricos, o estimador de densidade de Kernel, introduzido por Rosenblatt (1956), generaliza o histograma, usando uma função alternativa de pesos ${ }^{3}$. Deve destacar-se que os dados tiveram uma transformação logarítmica. A transformação logarítmica, uma das chamadas power transformations dentro da estatística, simplifica a estrutura dos dados, removendo distorções e outliers Bianchi (1997).

A análise das funções de densidade de Kernel sugere que, no ano de 1970, as economias se distribuíam em forma bimodal, mas já se vislumbrava o início de um terceiro grupo que aparece claramente em 1990 e no final do período analisado, no ano de 2003. A densidade apresenta certas características que devem ser notadas. Em primeiro lugar, vemos que a distribuição se modifica ao longo do tempo em duas dimensões, tanto na localização como na forma desta. Isto não é, no entanto, uma validação da inexistência de lock-in. Em presença de equilíbrios múltiplos, como parece ser este caso, vê-se em primeiro lugar um primeiro grupo de países - os mais pobres - que permanece na mesma situação desde a década de setenta: no trecho inicial da distribuição vemos que o limite inferior e a forma não mudam ao longo do tempo, só ficando mais achatada depois de 1970. Já no caso do grupo de países ricos, o deslocamento do último trecho da distribuição é evidente à medida que o tempo passa, o que comprovaria, dentro da teoria de path dependence à David (2003), que existe, para este grupo, um processo de "inovação exógena" - no estado do conhecimento relevante, ou no regime regulador institucional - que libera o sistema, fazendo-lo evoluir para uma nova configuração.

\footnotetext{
3 Esta função é dada por: $\hat{f}\left(x_{0}\right)=\frac{1}{N h} \sum_{i=1}^{N} K\left(\frac{x_{i}-x_{0}}{h}\right)$, onde o parâmetro h é o parâmetro de alisamento ou suavidade (smoothing parameter) chamado de amplitude. O K é o tipo de função de Kernel utilizada (Epanechnikov, Uniforme, Gaussiano, Quartic).
} 
Este tipo de análise, apesar de bastante intuitiva e esclarecedora, não nos informa o grau de confiança nas nossas conclusões sobre a existência dos clubes, os limites de suas abrangências, as médias de seus participantes, suas composições e principalmente não traz os movimentos inter-distribuição ao longo do tempo, o que poderá ser esclarecido a partir da análise de misturas finitas da distribuição. O emprego desta metodologia amplia as análises não-paramétricas em vários aspectos. A análise pioneira de clubes de convergência de Quah (1996) produz ricos resultados acerca de persistência e estratificação, formação de clubes de convergência, polarização da distribuição em países ricos e pobres. A análise a partir da metodologia de misturas finitas vai além, permitindo em primeiro lugar identificar, em forma mais apurada do que os métodos não-paramétricos, o número de grupos que existem dentro da distribuição. Em segundo lugar, possibilita conhecer quais são as médias e desvios padrão de cada um dos grupos que compõem a distribuição, como essas médias e erros padrão evoluem. O que permite identificar, por exemplo, se os países ricos são cada vez mais ricos e se os pobres caem em armadilhas de pobreza, permanecendo estancados no mesmo nível de renda ao longo do tempo. Uma outra contribuição desta metodologia é a fácil identificação dos países pertencentes a cada grupo e sua movimentação ao longo do tempo.

\section{Distribuição de misturas finitas: definição e Teste LR para o número de componentes}

Quando modelamos a distribuição da renda per capita dos países por uma mistura finita, determinar o número de componentes é um passo essencial da análise. A partir de um procedimento de inferência estatística, os dados por si podem determinar o número de componentes, e, com esse objetivo, uma mistura finita com componentes normais da distribuição de renda em log é uma ferramenta adequada.

Considere uma variável aleatória ou vetor aleatório $X$, tomando valores no espaço amostral $x \subset \mathfrak{R e}^{r}$, que pode ser discreta ou contínua, univariada ou multivariada. A distribuição de probabilidade da variável aleatória $X$ será caracterizada pela função de densidade de probabilidade. Seja $f_{X}$ a densidade de distribuição da renda per capita cross-country de um ano, e seja $f_{Y}$ a densidade da renda per capita, então $f_{Y}(y)=f_{X}\left(e^{y}\right) e^{y 4}$. A multimodalidade surge de $f_{X}$ como uma mistura finita de outras densidades unimodais, então:

$$
f_{X}(x)=p_{1} g\left(x ; \mu_{1}, \sigma_{1}\right)+\ldots+p_{m} g\left(x ; \mu_{m}, \sigma_{m}\right), \quad x>0
$$

em que $g_{i}(x)$ é a função de densidade das probabilidades para todo $i=1,2, \ldots, m$. A densidade univariada $g_{m}(x)$ é chamada de componente da densidade, e m é o número total de componentes. Os pesos $p_{i}>0, \sum p_{i}=1$. Neste caso, na distribuição de renda per capita cross-section, os componentes correspondem a grupos com diferentes níveis de renda per capita.

Chen et al. $(2001,2004)$ sugerem aplicar um teste modificado de razão de probabilidades (likelihood ratio test, LRT) para determinar o número de componentes do modelo de misturas finitas. Deve ser mencionado que este teste não é alterado sob transformação monotônica dos dados, sendo o resultado completamente consistente quando as variáveis são testadas em log.

\footnotetext{
${ }^{4}$ Determinar estatisticamente o número de componentes é o nosso objetivo, e esse número é preservado se os dados são transformados via transformação estritamente monotônica.
} 
Em primeiro lugar, se considera o teste de um sobre dois componentes no mix. Suponha que $\phi(x ; \mu, \sigma)$ é a distribuição normal com média $\mu$ e desvio padrão $\sigma$, e considere a mistura de dois componentes:

$$
f_{Y}\left(y ; p, \mu_{1}, \mu_{2}, \sigma\right)=p \phi\left(y ; \mu_{1}, \sigma\right)+(1-p) \phi\left(y ; \mu_{2}, \sigma\right)
$$

O problema a testar é $H_{0}: f_{Y}$ é normalmente distribuída contra $H_{1}: f_{Y}$ é de forma (2). A função verossimilhança é dada por:

$$
\begin{gathered}
l_{n}\left(p, \mu_{1}, \mu_{2}, \sigma\right)=\sum_{i=1}^{n} \log \left(p \phi\left(y_{i} ; \mu_{1}, \sigma\right)+(1-p) \phi\left(y_{i} ; \mu_{2}, \sigma\right)\right) \\
+C \log (4 p(1-p))
\end{gathered}
$$

onde $C$ é uma constante fixa (neste caso $C=2)$. Sejam $\left(\hat{p}, \hat{\mu}_{1}, \hat{\mu}_{2}, \hat{\sigma}\right)$ os parâmetros que maximizam $I_{n}\left(p, \mu_{1}, \mu_{2}, \sigma\right)$ e $(\hat{\mu}, \hat{\sigma})$ os que maximizam $I_{n}(1 / 2, \hat{\mu}, \hat{\mu}, \hat{\sigma})$. A hipótese $H_{1}$ é rejeitada para valores grandes da estatística LRT modificada:

$$
M_{n}=2\left(I_{n}\left(\hat{p}, \hat{\mu}_{1}, \hat{\mu}_{2}, \hat{\sigma}\right)-I_{n}(1 / 2, \hat{\mu}, \hat{\mu}, \hat{\sigma})\right)
$$

Para valores desconhecidos de $\sigma$ a distribuição assintótica de Mn é desconhecida; entretanto, Chen et al. (2001) demonstram que a distribuição $\chi_{2}^{2}$ (chi-quadrado) é uma boa aproximação no limite superior à distribuição assintótica de $M_{n}$.

No caso de testar dois contra mais componentes, o problema é: $H_{0}: f_{Y}$ é da forma (2) contra $H_{1}: f_{Y}$ tem mais de dois componentes. Podemos fixar o número de componentes sob a alternativa (no nosso caso, estimamos até $m=4$ ). Para uma mistura com $m$ componentes, o estimador de máxima verossimilhança (MLE), inserido na estatística LRT, é definido como o máximo de:

$$
\begin{array}{r}
l_{n}\left(\mu_{1}, \ldots, \mu_{m}, \sigma\right)=\sum_{i=1}^{n} \log \left(p_{1} \phi\left(y_{1} ; \mu_{1}, \sigma\right)+\ldots+p_{m} \phi\left(y_{m} ; \mu_{m}, \sigma\right)\right) \\
+C \log \left(\prod_{i-1}^{m} p_{i}\right)
\end{array}
$$

Neste caso, Chen et al. (2004) mostram que para um $\sigma$ conhecido, o teste LR modificado é assintoticamente distribuído como $q \chi_{0}^{2}+\frac{1}{2} \chi_{1}^{2}+(1-q) \chi_{2}^{2}$, onde a proporção q depende da distribuição mixing. Para um $\sigma$ desconhecido, a distribuição $\chi_{3}^{2}$ é o limite superior da distribuição assintótica.

\section{Resultados da analise econométrica}

\subsection{Definição dos grupos intra-distribuição}

Aplicamos a metodologia dos modelos de misturas finitas para o período de 1970 a 2003 para os 134 países do mundo. Na tabela 2, apresentam-se dois critérios de qualidade de ajuste dos modelos, quais sejam, o Critério de Informação de Akaike (AIC), (Akaike 1973) e o Critério de Informação Bayesiano (BIC) (Schwarz 1978), dados por $-2 l+2 k$ e $-2 l+k$, onde $l$ é o log da probabilidade, $k$ o número de parâmetros e $n$ o número de observações. 
Tabela 2: Critérios de Informação de Akaike e Schwartz para modelos de distintos números de componentes

\begin{tabular}{|c|c|c|c|c|c|c|c|c|c|}
\hline \multirow[b]{3}{*}{ Ano } & \multirow[b]{3}{*}{ Obs } & \multicolumn{8}{|c|}{ Número de componentes } \\
\hline & & \multicolumn{2}{|c|}{ Um } & \multicolumn{2}{|c|}{ Dois } & \multicolumn{2}{|c|}{ Três } & \multicolumn{2}{|c|}{ Quatro } \\
\hline & & AIC & BIC & AIC & BIC & AIC & BIC & AIC & BIC \\
\hline 1970 & 134 & 382,43 & 388,23 & 364,32 & 378,81 & 364.90 & 388,08 & - & - \\
\hline 1975 & 134 & 384,92 & 390,71 & 376,41 & 390,90 & 361,87 & 385,05 & 361,96 & 393,13 \\
\hline 1980 & 134 & 392,44 & 398,23 & 381,30 & 395,79 & 361,45 & 384,63 & 361,56 & 393,14 \\
\hline 1985 & 134 & 396,29 & 402,09 & 384,85 & 399,34 & 368,40 & 391,59 & 369,66 & 401,52 \\
\hline 1990 & 134 & 405,78 & 411,57 & 394,08 & 408,57 & 363,11 & 386,29 & 364,30 & 396,18 \\
\hline 1995 & 134 & 418,74 & 424,53 & 401,34 & 415,83 & 380,70 & 403,89 & 384,81 & 406,68 \\
\hline 2000 & 134 & 424,66 & 430,46 & 404,63 & 419,12 & 378,26 & 401,44 & 381,30 & 413,18 \\
\hline 2003 & 134 & 426,21 & 432,00 & 404,74 & 419,22 & 376,63 & 399,81 & 382,29 & 414,16 \\
\hline
\end{tabular}

${ }^{a}$ Fonte: Elaboração dos autores a partir dos resultados do Stata. 
Tabela 3: Teste LR - Dois grupos contra um, três contra dois, e quatro grupos contra três

\begin{tabular}{|c|c|c|c|c|c|c|c|}
\hline \multirow{2}{*}{ Ano } & \multirow{2}{*}{ Obs } & \multicolumn{2}{|c|}{ Dois contra um } & \multicolumn{2}{|c|}{ Três contra dois } & \multicolumn{2}{|c|}{ Quatro contra três } \\
\hline & & $\mathbf{L R} \chi^{2}$ & Prob. & $\overline{\operatorname{LR} \chi^{2}}$ & Prob. & $\operatorname{LR} \chi^{2}$ & Prob. \\
\hline 1970 & 134 & 24,11 & 0,0000 & 5,42 & 0,1435 & - & - \\
\hline 1975 & 134 & 14,51 & 0,0023 & 20,54 & 0,0001 & 6,61 & 0,0853 \\
\hline 1980 & 134 & 17,13 & 0,0007 & 25,85 & 0,0000 & 6,19 & 0,1028 \\
\hline 1985 & 134 & 17,44 & 0,0006 & 22,45 & 0,0001 & 4,76 & 0,1904 \\
\hline 1990 & 134 & 17,70 & 0,0005 & 36,97 & 0,0000 & 4,81 & 0,1865 \\
\hline 1995 & 134 & 23,39 & 0,0000 & 26,64 & 0,0000 & 3,90 & 0,1977 \\
\hline 2000 & 134 & 26,04 & 0,0000 & 32,36 & 0,0000 & 2,96 & 0,3979 \\
\hline 2003 & 134 & 27,47 & 0,0000 & 34,11 & 0,0000 & 0,34 & 0,9517 \\
\hline
\end{tabular}

${ }^{a}$ Fonte: Elaboração dos autores a partir dos resultados do Stata.

Deve ser lembrado que quanto menor o tamanho do AIC e BIC melhor o ajuste do modelo. Vemos que, para o ano inicial da amostra, 1970, ambos os critérios escolhem o modelo de dois componentes. A partir do ano de $1975 \mathrm{e}$ até o final da amostra, 2003, os critérios optam por três componentes dentro da amostra, respaldando a análise não-paramétrica.

Na tabela 3 são apresentados os resultados do teste LR mediante o qual se escolhe o número de grupos a partir de uma metodologia seqüencial, comparando primeiro um contra dois grupos, logo três contra dois; tendo isso sido necessário, senão podemos rejeitar a presença de três grupos, três contra quatro. As hipóteses nula e alternativa são dadas por: $H_{0}: f_{Y}$ têm $n$ componentes ${ }^{5}$ contra $H_{1}: f_{Y}$ tem $n+1$ componentes.

Como já foi mencionado, o teste LR convencional não é válido neste caso. Chen et al. (2004) sugerem, com base em estudo de simulação, sobre a hipótese nula de dois grupos, que a estatística calculada se distribui como uma $\chi^{2}$ com $2 v-2$ graus de liberdade, sendo $v$ o números de parâmetros extras no modelo de três grupos.

Em primeiro lugar podemos ver que sempre é preferível a hipótese de dois grupos à de um grupo dentro da distribuição. Isto já seria um questionamento às analises que utilizam dados de renda em forma homogênea para inferir convergência. É claro que, a partir deste resultado, a distribuição de renda por países apresenta no mínimo uma bimodalidade. Isto é, existe uma polarização na distribuição da renda e a forma de tratamento dos dados não poderia ser a partir de uma distribuição normal. A bimodalidade, todavia, é quebrada a partir do ano 1975, a partir do qual a presença de três grupos se materializa claramente.

A presença de grupos ou clubes de convergência a partir de estudos do tipo cross-section foi primeiramente salientada no trabalho Durlauf e Johnson (1995). Na sua contribuição, observam que a convergência global não se sustenta em amostras grandes porque os países pertencem a regimes diferentes, ou seja, grupos onde podemos, sim, esperar convergência, mas de tipo local. Os autores utilizam níveis iniciais de renda e de alfabetização para agrupar os países e encontrar as taxas de convergência dentro dos grupos, interpretando a heterogeneidade observada pelos parâmetros como indicativo da presença

\footnotetext{
${ }^{5}$ No caso de um componente, trata-se de uma distribuição normal.
} 
de regimes diferentes. A necessidade de selecionar critérios para agrupar países leva ao problema de como distinguir entre convergência em clubes e convergência condicional empiricamente. ${ }^{6}$ Este problema pode ser evitado pela utilização de modelos de mistura finita, dado que o agrupamento dos países acontece sem a necessidade da utilização de parâmetros complementares, como é observado neste caso.

Em suma, nossa análise confirma a hipótese levantada sob a análise gráfica, ou seja, de que no início da distribuição em 1970 temos a presença de dois grupos, mas que a partir de 1975 inicia-se uma configuração de 3 grupos que viria a ser confirmado nos anos seguintes, como observado pelo aumento de significância do teste LR sob hipótese nula de 3 grupos. A seguir, mostraremos as características básicas (média e desvio padrão) dos grupos de renda para cada ano, assim como a quantidade de países de cada grupo. Não podemos descartar que esta mudança de configuração dos grupos seja fruto de uma mudança macro-institucional que viria a modificar a economia política internacional. $\mathrm{O}$ início da década de 70 marca o final de uma era de ouro do capitalismo, com taxas de câmbio regulamentadas e alto crescimento da economia mundial. A mudança de estrutura do sistema financeiro mundial pode ter acarretado uma distribuição de renda entre os países distinta daquela observada no pós-guerra.

Observa-se principalmente uma maior dinâmica dos países intermediários, destacando-se estes daqueles mais pobres, enquanto o grupo de países ricos vê sua renda aumentar sem conhecer limites. Analisaremos estas dinâmicas na próxima seção, bem como identificaremos os componentes dos grupos e aqueles elementos que conseguiram em algum período migrar entre grupos.

\subsection{Composição dos grupos e evolução da distribuição}

A tabela 4 sumariza as principais características distribucionais dos modelos de dois componentes (1970) e três componentes (1975-2003): média $(u)$, erro padrão $(e p)$ e probabilidade de pertencer a cada grupo $(p)$.

O primeiro resultado importante da tabela é o estancamento ao longo do tempo da média do grupo de países pobres (em torno de US\$ 1.000 de renda per capita). Na passagem do ano 1970 a 1975, a queda pode ser atribuída à composição do novo grupo intermediário e a migração dos países com renda maior do grupo pobre para o grupo intermediário. Porém, o estancamento da média da renda per capita se mantém ao longo dos anos, ou seja, é clara a existência de uma "armadilha da pobreza" para este clube de renda. Enquanto ao numero de países pertencentes a este grupo, na primeira metade do período (1970-1990), produz-se uma queda constante.

A primeira queda importante, de 109 a 51 países é conseqüência da configuração do grupo de renda intermediária e da migração da metade dos países para esse novo grupo. No ano 1980, dois países africanos, Botsuana e Egito, e o Haiti, conseguem um aumento da renda suficiente para ingressar no grupo intermediário, mas o Haiti não consegue sustentar a posição e volta a cair para

\footnotetext{
${ }^{6}$ Como é salientado por Islam (2003), os determinantes de estado estacionário não podem ser usados para este propósito, dado que as diferenças nestes causam equilíbrios que diferem mesmo com baixa convergência condicional. Utilizar parâmetros time-varying (taxa de alfabetização ou níveis iniciais de renda) acarreta outros problemas.
} 
o grupo pobre no ano de 1985. No ano de 1985, Camarões, Cabo Verde, Congo e Honduras conseguem sair do grupo de renda baixa.

O ano de 1990 é aquele no qual mais países conseguem migrar deste grupo. Este resultado é associado a uma queda média e aumento do erro padrão do grupo intermediário mais do que a um aumento da renda dos países. Nesta situação, migram países como Costa do Marfim, Guiné, Haiti, Mongólia, Maldivas, Paquistão e Comores. A mudança da Índia e da Indonésia deve-se a um importante aumento da renda nesse período. No ano de 1995, com a queda do erro padrão do grupo intermediário, e a queda da renda, vários países, especialmente da África, não conseguem permanecer neste grupo: Camarões, Congo, Comores, Guiné, Haiti, Honduras, Mongólia, Paquistão e Índia aumentam seus níveis de renda, mas não o suficiente para se manter no grupo intermediário.

No ano de 2003, duas quedas do grupo intermediário aumentam o número de países pobres; trata-se dos casos de Bolívia e Zimbábue.

O grupo intermediário apresenta uma situação similar ao grupo pobre. A maior mudança acontece no ano de 1990 quando vários países do grupo de menor renda migram para este grupo e, logo, em 1995 voltam para o grupo um. Para este grupo, os resultados mostram também certo estancamento da média até 1990, uma queda no ano de 1995 e uma recuperação a partir do ano 2000. O erro padrão aumenta consideravelmente nos anos 1990, mas logo volta ao mesmo nível dos anos anteriores. A constância da dispersão de rendas per capita destes países nos permite inferir a inexistência de convergência de renda. Mesmo pertencendo a um mesmo grupo de renda, isso não significa que os países tenham um mesmo estado estacionário, apenas que são governados pelos mesmos processos estocásticos.

O grupo intermediário pode ser considerado o mais heterogêneo. Convivem nele países que têm sua renda quase no nível dos países mais ricos, como Portugal, Grécia, Hungria, Malásia, Omã, Coréia do Sul, com países latinoamericanos com rendas estancadas ao longo do tempo como Equador, El Salvador, Guatemala, Nicarágua. A renda per capita deste grupo passou de US\$ 4.359 em 1975, para US\$ 5.486 em 2003.

O grupo três, dos países ricos, tem aumento da média ao longo do período, e um erro padrão constante. Este resultado, unido ao já referido do grupo pobre é uma forte evidência do processo de ampliação da defasagem entre pobres e ricos no mundo: os países pobres mantêm a média de renda per capita em US\$ 1.000 ao longo de mais de 20 anos, mas os países ricos aumentam a sua média de US\$13.095, em 1970, para US\$23.155 no mesmo período.

A divergência das médias dos três grupos e as muito diferentes taxas de crescimento entre os grupos suportam a conclusão de convergência em grupos. Aem grupos. A renda do grupo rico quase dobrou de 1975 a 2003, a renda do grupo médio aumentou $25 \%$ aproximadamente e a renda do grupo pobre permaneceu estagnada, com uma pequena queda, o que mostra que o padrão de crescimento destes grupos é totalmente divergente.

Com respeito às percentagem de países pertencentes a cada grupo, todos apresentam pequena variação, se for considerado o ano inicial e final da amostra (e lembrando que no caso do grupo um, o ano 1970 representa o caso de dois grupos). No componente pobre, a percentagem de países cai de $38 \% \mathrm{em}$ 1975 para 35\% em 2003. O grupo de renda média quase não apresenta variação, representando $46 \%$ dos países em 1975 e indo para $44 \%$ em 2003 . O grupo rico ganhou países e a percentagem passou de 19\% para 21\% em 2003. 
Tabela 4: Média (ui), Erro Padrão (epi) e proporção (pi) de países por grupo (i)

\begin{tabular}{cccccccccc}
\hline Ano & $\mathbf{u 1}$ & ep1 & p1 $(\%)$ & u2 & ep2 & p2(\%) & u3 & ep3 & p3(\%) \\
\hline 1970 & 7,72 & 0,81 & 81,34 & - & - & - & 9,48 & 0,19 & 18,66 \\
1975 & 7,02 & 0,38 & 38,06 & 8,38 & 0,62 & 46,27 & 9,67 & 0,14 & 15,67 \\
1980 & 7,01 & 0,35 & 35,08 & 8,44 & 0,62 & 48,51 & 9,74 & 0,15 & 16,41 \\
1985 & 6,91 & 0,30 & 32,84 & 8,40 & 0,66 & 50,00 & 9,80 & 0,15 & 17,16 \\
1990 & 6,95 & 0,13 & 25,37 & 8,37 & 0,91 & 58,21 & 9,96 & 0,04 & 16,42 \\
1995 & 6,90 & 0,28 & 33,58 & 8,40 & 0,64 & 46,27 & 9,89 & 0,23 & 20,15 \\
2000 & 6,95 & 0,27 & 32,84 & 8,54 & 0,64 & 46,27 & 10,05 & 0,20 & 20,89 \\
2003 & 7,06 & 0,31 & 35,07 & 8,61 & 0,54 & 44,04 & 10,05 & 0,21 & 20,89 \\
\hline
\end{tabular}

${ }^{a}$ Fonte: Elaboração dos autores a partir dos resultados do Stata.

No Apêndice são apresentados todos os países componentes da amostra, assim como a evolução temporal das suas renda per capita. Alguns resultados devem ser salientados. Em primeiro lugar, dos países africanos que formam em sua maioria o grupo dos pobres, só alguns conseguem em algum momento do tempo migrar para o grupo intermediário (1985-1990), mas o fim do período os encontra a todos na mesma situação do começo.

Os países latino-americanos se encontram na sua maioria no grupo intermediário, alguns com aumento de renda ao longo do tempo (Chile, Brasil, República Dominicana, México, Panamá), a maioria com renda estancada ou crescimento muito baixo (Argentina, Equador, El Salvador, Guatemala, Jamaica, Paraguai, Peru, Uruguai, Venezuela) e outros com queda de renda e passagem para o grupo dos países pobres (Bolívia, Honduras, Nicarágua).

Os países asiáticos apresentam os melhores resultados. Taiwan, Singapura e Hong Kong conseguem migrar para o grupo de países ricos e sustentar a posição. Malásia e Coréia do Sul partem do grupo pobre e no final do período estão quase entrando no grupo de países ricos (sua renda per capita aumenta de US\$2.500 em 1970 para US\$12.088 em 2003 -Malásia - e US\$ 17.676 - Coréia do Sul-). Outros países como Laos, Coréia do Norte, Papua Nova Guiné, têm resultados menos expressivos, mas apresentam aumentos na sua renda também.

Resumindo, a evidência empírica suporta a existência de clubes e estratificação dos padrões do crescimento ao longo do tempo no mundo, em claro contraste com a hipótese de convergência. $\mathrm{O}$ mesmo resultado havia sido sugerido por Bianchi (1997), que encontra pequena mobilidade de países entre clubes, presença de convergência intra-clubes, aumento das diferenças entre países pobres e ricos entre os anos 1970 e 1980. Em síntese, forte evidência de bimodalidade numa amostra de 134 países no período 1970-1989. Porém, a análise do grupo intermediário se faz necessária dada a riqueza que o mesmo oferece em termos de estudo: trata-se de o grupo mais heterogêneo, onde convivem países que podem migrar tanto para o grupo pobre como para o grupo rico, e países que se perpetuam lá, na renda média.

A existência de equilíbrios múltiplos deixa em aberto duas questões David (2007). Primeiro: qual é a razão para supor a existência de mais de um equilíbrio? Isto requer examinar as estruturas existentes para demonstrar a plausibilidade do estado de equilíbrio contra-factual. Segundo: se outro estado de equilíbrio existe, como chegamos ao equilíbrio no qual nos encontramos? 
Isto requer identificar o mecanismo de "seleção", ou em termos de processos ramificados, analisar o processo de bifurcações críticas na seqüência do desenvolvimento e os fatores que condicionam as ações tomadas nas articulações históricas. A procura por uma reposta para estas perguntas excede os limites deste trabalho e ficam em aberto para pesquisas futuras.

\section{Observações finais}

Neste trabalho utilizamos o modelo de misturas finitas para pesquisar a distribuição de renda per capita de 134 países do mundo, o que permitiu fazer uma análise da dinâmica intra-distribuição a partir dos grupos determinados na estimação de misturas finitas, a probabilidade de pertencimento aos grupos, a evolução da média e o erro padrão e os regimes de transição entre datas selecionadas.

Em contraste com a literatura de convergência, os resultados mostram que só podemos inferir convergência em clubes. Mesmo assim, a análise de misturas finitas proporciona uma maior riqueza de resultados do que aqueles que podem ser conseguidos com o modelo de convergência condicional.

Nossa análise se enquadra na teoria de twin-peaks, especialmente nas idéias de existência de multimodalidade da distribuição, clubes de convergência, armadilhas de pobreza e riqueza, mas, em contraste a esta, encontramos evidência de um componente intermediário que emerge na metade da década de 1970, resultando numa distribuição de três componentes a partir de 1975. Isto é uma forte indicação de divergência dentro da distribuição. A diferença nos padrões de crescimento da renda entre os três grupos indicada pela evolução das médias de cada grupo é outro indicador desta divergência.

Para a existência de convergência condicional uma condição necessária é a redução da dispersão entre as economias. Não podemos observar esta condição em nenhum dos grupos analisados, em que esta, se não aumenta, permanece constante no período. Desta forma, os resultados que indicam convergência de renda per capita condicionada a valores iniciais de disponibilidade de capital físico e humano podem ser considerados como em Islam (2003) e Durlauf e Johnson (1995), como resultado de uma regressão à média, e as conclusões são, portanto, espúrias.

Não podemos descartar que o ambiente institucional mundial exerça um papel fundamental nesta distribuição. A mudança de configuração do número de grupos, juntamente com as constantes mudanças de probabilidades de transição entre os diferentes grupos, sugere que existem mudanças não consideradas em nossa análise que podem alterar a economia política internacional. A partir da década de 90, data de intensificação do comércio internacional, observa-se um interessante caso de armadilha da riqueza, onde as economias que atingem o estado mais rico ficam "presas" sem ser observadas mudanças em direção ao grupo de renda intermediária. O ano de término do tratado de Bretton Woods também marca uma importante mudança na configuração dos grupos, abrindo espaço para o crescimento de um grupo intermediário de renda e deixando para trás um grupo de países pobres com a renda estagnada.

Em suma, podemos dizer que, ao longo de mais de 30 anos, o status quo predomina e são claros os ganhadores e perdedores na questão da distribuição de renda per capita dos distintos países do mundo. 



\section{Apêndice A}

Países e sua evolução temporal da renda

RB: renda baixa

RM: renda média

RA: renda alta

\begin{tabular}{|c|c|c|c|c|c|c|c|c|}
\hline País & 1970 & 1975 & 1980 & 1985 & 1990 & 1995 & 2000 & 2003 \\
\hline Benin & $\mathrm{RB}$ & $\mathrm{RB}$ & $\mathrm{RB}$ & $\mathrm{RB}$ & $\mathrm{RB}$ & $\mathrm{RB}$ & $\mathrm{RB}$ & $\mathrm{RB}$ \\
\hline Bolívia & $\mathrm{RB}$ & RM & RM & RM & RM & RM & RM & RB \\
\hline Burkina Faso & $\mathrm{RB}$ & $\mathrm{RB}$ & $\mathrm{RB}$ & $\mathrm{RB}$ & $\mathrm{RB}$ & $\mathrm{RB}$ & $\mathrm{RB}$ & $\mathrm{RB}$ \\
\hline Burundi & $\mathrm{RB}$ & $\mathrm{RB}$ & $\mathrm{RB}$ & $\mathrm{RB}$ & $\mathrm{RB}$ & $\mathrm{RB}$ & $\mathrm{RB}$ & $\mathrm{RB}$ \\
\hline Camarões & $\mathrm{RB}$ & $\mathrm{RB}$ & $\mathrm{RB}$ & RM & $\mathrm{RM}$ & RB & $\mathrm{RB}$ & $\mathrm{RB}$ \\
\hline África Central & $\mathrm{RB}$ & $\mathrm{RB}$ & $\mathrm{RB}$ & $\mathrm{RB}$ & RM & RB & $\mathrm{RB}$ & $\mathrm{RB}$ \\
\hline Chade & $\mathrm{RB}$ & $\mathrm{RB}$ & $\mathrm{RB}$ & $\mathrm{RB}$ & RM & RB & $\mathrm{RB}$ & $\mathrm{RB}$ \\
\hline Comores & $\mathrm{RB}$ & $\mathrm{RB}$ & $\mathrm{RB}$ & $\mathrm{RB}$ & RM & RB & $\mathrm{RB}$ & $\mathrm{RB}$ \\
\hline Congo & $\mathrm{RB}$ & $\mathrm{RB}$ & $\mathrm{RB}$ & RM & RM & RB & $\mathrm{RB}$ & $\mathrm{RB}$ \\
\hline Costa do Marfim & $\mathrm{RB}$ & $\mathrm{RB}$ & $\mathrm{RB}$ & $\mathrm{RB}$ & RM & $\mathrm{RB}$ & $\mathrm{RB}$ & $\mathrm{RB}$ \\
\hline Etiópia & $\mathrm{RB}$ & $\mathrm{RB}$ & $\mathrm{RB}$ & $\mathrm{RB}$ & $\mathrm{RB}$ & $\mathrm{RB}$ & $\mathrm{RB}$ & $\mathrm{RB}$ \\
\hline Gâmbia & $\mathrm{RB}$ & $\mathrm{RB}$ & $\mathrm{RB}$ & $\mathrm{RB}$ & $\mathrm{RB}$ & $\mathrm{RB}$ & $\mathrm{RB}$ & $\mathrm{RB}$ \\
\hline Gana & $\mathrm{RB}$ & $\mathrm{RB}$ & $\mathrm{RB}$ & $\mathrm{RB}$ & $\mathrm{RB}$ & $\mathrm{RB}$ & $\mathrm{RB}$ & $\mathrm{RB}$ \\
\hline Guiné & $\mathrm{RB}$ & RM & RM & RB & RM & RB & $\mathrm{RB}$ & $\mathrm{RB}$ \\
\hline Haiti & $\mathrm{RB}$ & $\mathrm{RB}$ & RM & RB & RM & RB & $\mathrm{RB}$ & $\mathrm{RB}$ \\
\hline Honduras & $\mathrm{RB}$ & $\mathrm{RB}$ & $\mathrm{RB}$ & RM & RM & RB & $\mathrm{RB}$ & $\mathrm{RB}$ \\
\hline Índia & $\mathrm{RB}$ & $\mathrm{RB}$ & $\mathrm{RB}$ & $\mathrm{RB}$ & RM & RB & $\mathrm{RB}$ & $\mathrm{RB}$ \\
\hline Iraque & $\mathrm{RB}$ & RM & RM & RM & RM & RB & $\mathrm{RB}$ & $\mathrm{RB}$ \\
\hline Quênia & $\mathrm{RB}$ & $\mathrm{RB}$ & $\mathrm{RB}$ & $\mathrm{RB}$ & $\mathrm{RB}$ & $\mathrm{RB}$ & $\mathrm{RB}$ & $\mathrm{RB}$ \\
\hline Kiribati & $\mathrm{RB}$ & RM & RB & $\mathrm{RB}$ & $\mathrm{RB}$ & $\mathrm{RB}$ & $\mathrm{RB}$ & $\mathrm{RB}$ \\
\hline Coréia, Dem. Rep. & $\mathrm{RB}$ & $\mathrm{RB}$ & $\mathrm{RB}$ & $\mathrm{RB}$ & $\mathrm{RB}$ & $\mathrm{RB}$ & $\mathrm{RB}$ & $\mathrm{RB}$ \\
\hline Laos & $\mathrm{RB}$ & $\mathrm{RB}$ & $\mathrm{RB}$ & $\mathrm{RB}$ & $\mathrm{RB}$ & $\mathrm{RB}$ & $\mathrm{RB}$ & $\mathrm{RB}$ \\
\hline Lesotho & $\mathrm{RB}$ & $\mathrm{RB}$ & $\mathrm{RB}$ & $\mathrm{RB}$ & $\mathrm{RB}$ & $\mathrm{RB}$ & $\mathrm{RB}$ & $\mathrm{RB}$ \\
\hline Madagascar & $\mathrm{RB}$ & $\mathrm{RB}$ & $\mathrm{RB}$ & $\mathrm{RB}$ & $\mathrm{RB}$ & $\mathrm{RB}$ & $\mathrm{RB}$ & $\mathrm{RB}$ \\
\hline Malawi & $\mathrm{RB}$ & $\mathrm{RB}$ & $\mathrm{RB}$ & $\mathrm{RB}$ & $\mathrm{RB}$ & $\mathrm{RB}$ & $\mathrm{RB}$ & $\mathrm{RB}$ \\
\hline Mali & $\mathrm{RB}$ & $\mathrm{RB}$ & $\mathrm{RB}$ & $\mathrm{RB}$ & $\mathrm{RB}$ & $\mathrm{RB}$ & $\mathrm{RB}$ & $\mathrm{RB}$ \\
\hline Mauritânia & $\mathrm{RB}$ & $\mathrm{RB}$ & $\mathrm{RB}$ & $\mathrm{RB}$ & $\mathrm{RB}$ & $\mathrm{RB}$ & $\mathrm{RB}$ & $\mathrm{RB}$ \\
\hline Mongólia & $\mathrm{RB}$ & $\mathrm{RB}$ & $\mathrm{RB}$ & $\mathrm{RB}$ & $\mathbf{R M}$ & RB & $\mathrm{RB}$ & $\mathrm{RB}$ \\
\hline Moçambique & $\mathrm{RB}$ & $\mathrm{RB}$ & $\mathrm{RB}$ & $\mathrm{RB}$ & $\mathrm{RB}$ & $\mathrm{RB}$ & $\mathrm{RB}$ & $\mathrm{RB}$ \\
\hline Nepal & $\mathrm{RB}$ & $\mathrm{RB}$ & $\mathrm{RB}$ & $\mathrm{RB}$ & $\mathrm{RB}$ & $\mathrm{RB}$ & $\mathrm{RB}$ & $\mathrm{RB}$ \\
\hline Niger & $\mathrm{RB}$ & $\mathrm{RB}$ & $\mathrm{RB}$ & $\mathrm{RB}$ & $\mathrm{RB}$ & $\mathrm{RB}$ & $\mathrm{RB}$ & $\mathrm{RB}$ \\
\hline Nigéria & $\mathrm{RB}$ & $\mathrm{RB}$ & $\mathrm{RB}$ & $\mathrm{RB}$ & $\mathrm{RB}$ & $\mathrm{RB}$ & $\mathrm{RB}$ & $\mathrm{RB}$ \\
\hline Paquistão & $\mathrm{RB}$ & $\mathrm{RB}$ & $\mathrm{RB}$ & $\mathrm{RB}$ & $\mathbf{R M}$ & RB & $\mathrm{RB}$ & $\mathrm{RB}$ \\
\hline Ruanda & $\mathrm{RB}$ & $\mathrm{RB}$ & $\mathrm{RB}$ & $\mathrm{RB}$ & $\mathrm{RB}$ & $\mathrm{RB}$ & $\mathrm{RB}$ & $\mathrm{RB}$ \\
\hline São Tomé e Príncipe & $\mathrm{RB}$ & $\mathrm{RB}$ & $\mathrm{RB}$ & $\mathrm{RB}$ & $\mathrm{RB}$ & $\mathrm{RB}$ & $\mathrm{RB}$ & $\mathrm{RB}$ \\
\hline Senegal & $\mathrm{RB}$ & $\mathrm{RB}$ & $\mathrm{RB}$ & $\mathrm{RB}$ & $\mathrm{RB}$ & $\mathrm{RB}$ & $\mathrm{RB}$ & $\mathrm{RB}$ \\
\hline Serra Leoa & $\mathrm{RB}$ & $\mathrm{RB}$ & $\mathrm{RB}$ & $\mathrm{RB}$ & $\mathrm{RB}$ & $\mathrm{RB}$ & $\mathrm{RB}$ & $\mathrm{RB}$ \\
\hline Ilhas Salomão & $\mathrm{RB}$ & $\mathrm{RB}$ & $\mathrm{RB}$ & $\mathrm{RB}$ & RM & RM & RB & $\mathrm{RB}$ \\
\hline
\end{tabular}




\begin{tabular}{|c|c|c|c|c|c|c|c|c|}
\hline País & 1970 & 1975 & 1980 & 1985 & 1990 & 1995 & 2000 & 2003 \\
\hline Somália & $\mathrm{RB}$ & $\mathrm{RB}$ & $\mathrm{RB}$ & $\mathrm{RB}$ & $\mathrm{RB}$ & $\mathrm{RB}$ & $\mathrm{RB}$ & $\mathrm{RB}$ \\
\hline Sudão & RB & RB & RB & RB & RB & $\mathrm{RB}$ & $\mathrm{RB}$ & RB \\
\hline Syrian Arab Republic & $\mathrm{RB}$ & $\mathrm{RB}$ & $\mathrm{RB}$ & $\mathrm{RB}$ & $\mathrm{RB}$ & $\mathrm{RB}$ & $\mathrm{RB}$ & $\mathrm{RB}$ \\
\hline Tanzânia & $\mathrm{RB}$ & $\mathrm{RB}$ & $\mathrm{RB}$ & $\mathrm{RB}$ & $\mathrm{RB}$ & $\mathrm{RB}$ & $\mathrm{RB}$ & $\mathrm{RB}$ \\
\hline Togo & $\mathrm{RB}$ & $\mathrm{RB}$ & $\mathrm{RB}$ & $\mathrm{RB}$ & $\mathrm{RB}$ & $\mathrm{RB}$ & $\mathrm{RB}$ & $\mathrm{RB}$ \\
\hline Uganda & $\mathrm{RB}$ & $\mathrm{RB}$ & $\mathrm{RB}$ & $\mathrm{RB}$ & $\mathrm{RB}$ & $\mathrm{RB}$ & $\mathrm{RB}$ & $\mathrm{RB}$ \\
\hline Vanuatu & $\mathrm{RB}$ & $\mathbf{R M}$ & RM & RM & RM & $\mathrm{RM}$ & RM & RB \\
\hline Zâmbia & $\mathrm{RB}$ & $\mathrm{RB}$ & $\mathrm{RB}$ & $\mathrm{RB}$ & RM & $\mathrm{RB}$ & $\mathrm{RB}$ & $\mathrm{RB}$ \\
\hline Zimbábue & $\mathrm{RB}$ & RM & RM & RM & RM & RM & RM & RB \\
\hline Algéria & $\mathrm{RB}$ & RM & RM & RM & RM & RM & RM & RM \\
\hline Antígua e Barbuda & $\mathrm{RB}$ & RM & $\mathrm{RM}$ & RM & RM & RM & RM & RM \\
\hline Argentina & RA & RM & RM & $\mathrm{RM}$ & $\mathrm{RM}$ & RM & RM & $\mathrm{RM}$ \\
\hline Bahamas & RA & RM & RA & RA & RA & RA & RA & RM \\
\hline Barbados & RA & RM & RM & RM & RM & RM & RM & RM \\
\hline Belize & $\mathrm{RB}$ & RM & RM & RM & RM & RM & RM & RM \\
\hline Botsuana & $\mathrm{RB}$ & $\mathrm{RB}$ & RM & RM & RM & RM & RM & RM \\
\hline Brasil & $\mathrm{RB}$ & RM & RM & RM & RM & RM & RM & RM \\
\hline Cabo Verde & $\mathrm{RB}$ & $\mathrm{RB}$ & $\mathrm{RB}$ & RM & RM & RM & RM & RM \\
\hline Chile & $\mathrm{RB}$ & RM & RM & RM & RM & RM & RM & RM \\
\hline China & $\mathrm{RB}$ & $\mathrm{RB}$ & $\mathrm{RB}$ & $\mathrm{RB}$ & $\mathrm{RB}$ & RM & RM & RM \\
\hline Colômbia & $\mathrm{RB}$ & RM & RM & RM & RM & RM & RM & RM \\
\hline Costa Rica & $\mathrm{RB}$ & RM & RM & RM & RM & RM & RM & RM \\
\hline Cuba & $\mathrm{RB}$ & RM & RM & RM & RM & RM & RM & RM \\
\hline Dominica & $\mathrm{RB}$ & RM & RM & RM & RM & $\mathrm{RM}$ & $\mathrm{RM}$ & $\mathrm{RM}$ \\
\hline República Dominicana & $\mathrm{RB}$ & $\mathbf{R M}$ & RM & RM & RM & RM & RM & RM \\
\hline Equador & $\mathrm{RB}$ & RM & RM & RM & RM & RM & RM & RM \\
\hline Egito. & $\mathrm{RB}$ & $\mathrm{RB}$ & RM & RM & RM & RM & RM & RM \\
\hline El Salvador & $\mathrm{RB}$ & $\mathbf{R M}$ & RM & RM & RM & RM & RM & RM \\
\hline Guiné Equatorial & $\mathrm{RB}$ & $\mathrm{RB}$ & $\mathrm{RB}$ & $\mathrm{RB}$ & $\mathrm{RB}$ & $\mathrm{RB}$ & RM & RM \\
\hline Fiji & $\mathrm{RB}$ & RM & RM & RM & RM & RM & RM & RM \\
\hline Gabão & RA & RA & RA & RM & RM & RM & RM & RM \\
\hline Grécia & $\mathrm{RB}$ & RM & $\mathrm{RM}$ & RM & RM & RM & RM & RM \\
\hline Granada & $\mathrm{RB}$ & RM & $\mathrm{RM}$ & RM & RM & $\mathrm{RM}$ & RM & RM \\
\hline Guatemala & $\mathrm{RB}$ & RM & RM & RM & RM & RM & RM & RM \\
\hline Hungria & $\mathrm{RB}$ & RM & RM & RM & RM & RM & RM & RM \\
\hline Indonésia & $\mathrm{RB}$ & $\mathrm{RB}$ & $\mathrm{RB}$ & $\mathrm{RB}$ & RM & RM & RM & RM \\
\hline Irã & $\mathrm{RB}$ & RM & RM & RM & RM & RM & RM & RM \\
\hline Jamaica & $\mathrm{RB}$ & RM & $\mathrm{RM}$ & RM & RM & RM & RM & RM \\
\hline Jordânia & $\mathrm{RB}$ & RM & RM & RM & RM & RM & RM & RM \\
\hline Coréia. & $\mathrm{RB}$ & RM & RM & $\mathrm{RM}$ & RM & RM & RM & RM \\
\hline Malásia & $\mathrm{RB}$ & RM & RM & $\mathrm{RM}$ & RM & RM & RM & RM \\
\hline Maldivas & $\mathrm{RB}$ & $\mathrm{RB}$ & $\mathrm{RB}$ & $\mathrm{RB}$ & RM & $\mathrm{RM}$ & RM & RM \\
\hline Maurício & $\mathrm{RB}$ & RM & RM & RM & RM & RM & RM & RM \\
\hline México & $\mathrm{RB}$ & RM & RM & RM & RM & RM & RM & RM \\
\hline Marrocos & $\mathrm{RB}$ & RM & RM & RM & RM & $\mathrm{RM}$ & RM & $\mathrm{RM}$ \\
\hline Namíbia & $\mathrm{RB}$ & RM & $\mathrm{RM}$ & RM & RM & RM & RM & RM \\
\hline Nicarágua & $\mathrm{RB}$ & RM & RM & RM & RM & RM & RM & RM \\
\hline Omã & RA & RM & RM & RM & RM & RM & RM & RM \\
\hline Panamá & $\mathrm{RB}$ & RM & RM & $\mathrm{RM}$ & RM & RM & RM & RM \\
\hline Papua Nova Guiné & $\mathrm{RB}$ & RM & $\mathrm{RM}$ & $\mathrm{RM}$ & RM & RM & RM & RM \\
\hline Paraguai & $\mathrm{RB}$ & RM & RM & RM & RM & RM & RM & RM \\
\hline Peru & $\mathrm{RB}$ & RM & RM & RM & RM & RM & RM & RM \\
\hline Filipinas & $\mathrm{RB}$ & RM & RM & $\mathrm{RM}$ & RM & RM & $\mathrm{RM}$ & $\mathrm{RM}$ \\
\hline Polônia & $\mathrm{RB}$ & RM & $\mathrm{RM}$ & RM & RM & RM & RM & RM \\
\hline Portugal & $\mathrm{RB}$ & RM & $\mathrm{RM}$ & RM & RM & RM & RM & RM \\
\hline Romênia & $\mathrm{RB}$ & RM & RM & RM & RM & RM & RM & RM \\
\hline
\end{tabular}




\begin{tabular}{|c|c|c|c|c|c|c|c|c|}
\hline País & 1970 & 1975 & 1980 & 1985 & 1990 & 1995 & 2000 & 2003 \\
\hline Samoa & $\mathrm{RB}$ & $\mathbf{R M}$ & RM & RM & RM & $\mathrm{RB}$ & $\mathrm{RM}$ & $\mathrm{RM}$ \\
\hline África do Sul & $\mathrm{RB}$ & $\mathbf{R M}$ & RM & RM & $\mathrm{RM}$ & RM & $\mathrm{RM}$ & RM \\
\hline Sri Lanka & $\mathrm{RB}$ & $\mathrm{RB}$ & $\mathrm{RB}$ & $\mathbf{R M}$ & $\mathrm{RM}$ & $\mathrm{RM}$ & $\mathrm{RM}$ & RM \\
\hline Suriname & $\mathrm{RB}$ & RM & RM & RM & RM & RM & $\mathrm{RM}$ & RM \\
\hline Suazilândia & $\mathrm{RB}$ & RM & RM & $\mathrm{RM}$ & $\mathrm{RM}$ & $\mathrm{RM}$ & RM & RM \\
\hline Tailândia & $\mathrm{RB}$ & $\mathrm{RB}$ & RM & RM & RM & RM & RM & RM \\
\hline Tonga & $\mathrm{RB}$ & $\mathrm{RB}$ & $\mathrm{RB}$ & $\mathbf{R M}$ & $\mathrm{RM}$ & $\mathrm{RM}$ & RM & RM \\
\hline Trinidad e Tobago & $\mathrm{RB}$ & RM & RM & RM & RM & RM & RM & RM \\
\hline Tunísia & $\mathrm{RB}$ & $\mathbf{R M}$ & RM & $\mathrm{RM}$ & RM & $\mathrm{RM}$ & RM & $\mathrm{RM}$ \\
\hline Turquia & $\mathrm{RB}$ & RM & RM & RM & RM & RM & RM & RM \\
\hline Uruguai & $\mathrm{RB}$ & RM & RM & RM & RM & RM & $\mathrm{RM}$ & RM \\
\hline Venezuela, RB & $\mathrm{RB}$ & RM & RM & RM & RM & RM & $\mathrm{RM}$ & RM \\
\hline Austrália & RA & RA & RA & RA & RA & RA & RA & RA \\
\hline Áustria & RA & RA & RA & RA & RA & RA & RA & RA \\
\hline Bahrain & RA & RA & RA & RA & RM & RA & RA & RA \\
\hline Bélgica & RA & RA & RA & RA & RA & RA & RA & RA \\
\hline Canadá & RA & RA & RA & RA & RA & RA & RA & RA \\
\hline Chipre & $\mathrm{RB}$ & RM & RM & RM & $\mathrm{RM}$ & RA & RA & RA \\
\hline Dinamarca & RA & RA & RA & RA & RA & RA & RA & RA \\
\hline Finlândia & RA & RA & RA & RA & RA & RA & RA & RA \\
\hline França & RA & RA & RA & RA & RA & RA & RA & RA \\
\hline Alemanha & RA & RA & RA & RA & RA & RA & RA & RA \\
\hline Hong Kong, China & $\mathrm{RB}$ & RM & RM & RA & RA & RA & RA & RA \\
\hline Islândia & RA & RA & RA & RA & RA & RA & RA & RA \\
\hline Irlanda & $\mathrm{RB}$ & RM & $\mathrm{RM}$ & RM & $\mathrm{RM}$ & RA & RA & RA \\
\hline Israel & RA & RA & RA & RA & RA & RA & RA & RA \\
\hline Itália & RA & RA & RA & RA & RA & RA & RA & RA \\
\hline Japão & RA & RA & RA & RA & RA & RA & RA & RA \\
\hline Malta & $\mathrm{RB}$ & RM & $\mathrm{RM}$ & RM & $\mathrm{RM}$ & $\mathrm{RM}$ & $\mathrm{RM}$ & RA \\
\hline Holanda & RA & RA & RA & RA & RA & RA & RA & RA \\
\hline Nova Zelândia & RA & RA & RA & RA & RA & RA & RA & RA \\
\hline Noruega & RA & RA & RA & RA & RA & RA & RA & RA \\
\hline Porto Rico & $\mathrm{RB}$ & RM & $\mathrm{RM}$ & RM & $\mathrm{RM}$ & RA & RA & RA \\
\hline Singapura & $\mathrm{RB}$ & $\mathbf{R M}$ & RM & RA & RA & RA & RA & RA \\
\hline Espanha & $\mathrm{RB}$ & $\mathbf{R M}$ & RM & RM & RM & RA & RA & RA \\
\hline Suécia & RA & RA & RA & RA & RA & RA & RA & RA \\
\hline Suíça & RA & RA & RA & RA & RA & RA & RA & RA \\
\hline Taiwan & $\mathrm{RB}$ & RM & $\mathrm{RM}$ & $\mathrm{RM}$ & $\mathrm{RM}$ & $\mathrm{RM}$ & RA & RA \\
\hline Reino Unido & RA & RA & RA & RA & RA & RA & RA & RA \\
\hline Estados Unidos & RA & RA & RA & RA & RA & RA & RA & RA \\
\hline
\end{tabular}

\section{Referências Bibliográficas}

A. Ades e H. Chua. The neighbor's curse: regional instability and economic growth. Journal of Economic Growth, 2:279-304, 1997.

H. Akaike. Information theory and an extension of the maximum likelihood principle. In B. N. Petrov e P. Csaki, editores, Second International Symposium on Information Theory, pages 267281, 1973.

A. Alesina, S. Ozler, N. Roubini, e P. Swagel. Political instability and economic growth. Journal of Economic Growth, 1:189-211, 1996.

R. Barro. Economic growth in a cross section of countries. NBER, working paper, 1991.

R. Barro e X. Sala-I-Martin. Economic growth and convergence across the united states. Technical report, NBER, 1990.

R. Barro e X. Sala-I-Martin. Convergence. Journal of Political Economy, 100:223-251, 1992.

W. J. Baumol. Productivity gorwth, convergence and welfare: what the long-run data show. American Economic Review of Economic Studies, 76:1072-1085, 1986. 
D. Ben-David e M. Loewy. Free trade growth and convergence. Journal of Economic Growth, 3: 143-170, 1998.

J. Benhabib e A. Rustichini. Social conflict and gorwth. Journal of Economic Growth, 1:125-142, 1996.

M. Bianchi. Testing for convergence: evidence from non-parametric multimodality test. Journal of Applied Econometrics, 12:393-409, 1997.

F. Castellacci e D. Archibugi. The technology clubs in the world economy. mimeo. Mimeo, 2005.

H. Chen, J. Chen, e J. D. Kalbfleisch. A modified likelihood ratio test for homogeneity in finite mixture models. Journal of Royal Statistical Society Serie B: Statistical Methodology, 63:19-29, 2001.

H. Chen, J. Chen, e J. D. Kalbfleisch. Testing for a finite mixture model with two components. Journal of Royal Statistical Society Serie B: Statistial Methodology, 66:95-115, 2004.

P. A. David. Path dependence, its critics and the quest for "historical economics". Economic History, 50, 2003.

P. A. David. Path dependence: a foundational concept for historical science. Cliometrica, 1: 91-114, 2007.

D. Dollar e E. Wolff. Competitiveness, convergence, and international specialization. The MIT Press, 1994.

S. N. Durlauf e P. A. Johnson. Multip0le regimes and cross-country growth behavior. Journal of Applied Econometrics, 10:365-384, 1995.

P. Evans. How fast do economics converge. Review of Economics and Statistics, 79:219-225, 1997.

S. Frühwirth-Schnatter. Finite mixture and markov switching models. New York: Springer, 2006.

O. Galor e D. Tsiddon. The distribuition of human capital and economic growth. Journal of Economic Growth, 2:93-124, 1997.

A. Heston, R. Summers, e B. Aten. Penn world table version 6.2. Technical report, Center for International Comparisons of Production, Income and Prices at the University of Pennsylvania, http://pwt.econ.upenn.edu/, september 2006.

P. Howitt e D. Mayer-Foulkes. Implementation and stagnation: a schumpeterian theory of convergence clubs. Master's thesis, NBER, 2002.

N. Islam. What have we learnt from the convergence debate? Journal of Economic Surveys, 17: 309-362, 2003.

J. W. Lee. Government interventions and productivity growth. Journal of Economic Growth, 1: 391-414, 1996.

R. Levine e D. Renelt. A sebsitivity analysis of cross-country growth regressions. American Economic Review, 82:942-963, 1992.

R. Lucas. On the machanics of economic development. Journal of Monetary Economics, 22:3-42, 1988.

N. Mankiw, D. Romer, e D. Weil. A contribution to the empirics of economic growth. Quartely Journal of Economics, 107:207-237, 1992.

R. Moreno e B. Trehan. Location and the growth of nations. Journal of Economic Growth, 2: 399-418, 1997.

T. Nakajima. Cath-up in turn in a multi-country international trade model learning-by-doing and inventation. Journal of Development Economics, 72:117-138, 2003.

D. Quah. Empirics for growth and distribuition: stratification, polarization, and convergence clubs. Journal of Economics Growth, 2:27-59, 1997.

D. T. Quah. Empirical cross-section dynamics in economic growth. European Economic Review, 37:426-434, 1993.

D. T. Quah. Twin peaks: growth and convergence in models of distribution dynamics. Economic Journal, 106:1045-1055, 1996. 
P. Romer. Increasing returns and long run growth. Journal of Political Economy, 94:1002-1037, 1986.

M. Rosenblatt. Remarks on some nonparametric estimates of a density function. Annals of Mathematical Statistics, 27:832-837, 1956.

G. Schwarz. Estimating the dimension of a model. Annals of Statistics, 6:461-464, 1978.

R. Solow. A contribution to the theory of economics growth. Quartely Journal of Economics, 70: 65-94, 1956. 